\title{
Eva Jeppsson Grassman and Anna Whitaker (eds.) (2013). Ageing with Disability. A Lifecourse Perspective. Bristol: Policy Press, 142 pp. ISBN 9781447305224 (hardback)
}

\author{
REVIEWED by VALERIE $D^{\prime}$ ASTOUS ${ }^{*}$
}

\begin{abstract}
Ageing and long-term disability do not generally overlap in current theory, policy or practice leaving the issues of and people with these experiences poorly recognised, understood and supported. Ageing with Disability: A Lifecourse Perspective, edited by Jeppsson Grassman and Whitaker, is a timely and valuable contribution to the ageing and disability discourse. It provides a vital, critical context to increase understanding and awareness of the unique experiences of individuals ageing with a disability acquired in early life. This perspective is of substantial importance for many students, academics, professionals, advocates and policy makers. The editors and contributing authors to this text have expertise and research experience in the fields of disability, ageing and social policy. The aim of the book is to discuss from a lifecourse perspective what it means to live and age with a long-term disability. The text is punctuated by subjective accounts that highlight the implications, challenges and changes over time both for the individual and within social and political environments. A comprehensive scope of ageing with long-term physical and mental disabilities, and a diversity of caregiving roles are presented. These are framed within the lifecourse perspective and so contest current concepts of successful ageing, confront limitations in policy and practice, and inform gaps in knowledge.
\end{abstract}

*Valerie D'Astous, Institute of Gerontology, King's College London, London, UK 
International Journal of Ageing and Later Life

The editors bracket contributor chapters with an introduction to create the platform and a summary and conclusion to reiterate key points and emphasise the need for continued research. Chapter two by Jeppsson Grassman outlines a life trajectory with chronic illness and disability where bodily changes, cumulative impairments and complications shape lives and life experiences of ageing. It accentuates a life of constant adaptations to new challenges and disablements across the lifecourse. Chapters three and four by Holme and Taghizadeh Larsson respectively highlight how early life stages with a long-term disability affect later life, as do changes in the social context, environmental accommodations and policy over time. They challenge and explore the potential of successful ageing with a disability or impairment. With effective environmental, financial and supportive conditions individuals ageing with a long-term physical disability are able to maintain and achieve an active, engaged, self-realising lifestyle. In contrast, chapter five, by Bulow and Svensson, describes a lifecourse of decreased engagement and socially isolated lives prescribed by long-term mental disabilities as not compatible with successful ageing. Chapters six and seven (Whitaker and Torge) frame the experiences of ageing and caregiving for loved ones with long-term disabilities from the perspectives of a parent and a partner. The lasting responsibilities, balance and imbalance, and acquired expertise over the years are permeated by worry, effort and love.

The organization, not just on the chapter level but also in the threading of the lifecourse perspective of ageing with disability throughout the text, is noteworthy and underscores distinct aspects. For example, in a long life characterised by mental and physical disability, chronological age and advancing years hold varied meaning, and are perceived and experienced differently. Moreover, time is often marked by the length of the experience of disability or chronic illness, or from timeframes between complications or treatments. As age norms are replaced with illness norms, a heightened awareness of the possibility of a shortened life referenced by that of peers appears to be commonly experienced. Policy and social changes acknowledge advancements and detailed outstanding disabling barriers.

This book presents a missing perspective in the discourse of ageing and disability. Drawing on a wealth of longitudinal and cross-sectional qualitative research with a strong sense of narrative, the chapters in this 
book clearly articulate the intersections and interrelatedness of ageing with a long-term disability or chronic illness. However, greater detail to the overall structure of the book may have created a more fluid and welldefined representation of key themes and arguments. The chapters often seem disjointed with no linking statements or transitions. A reordering of the chapters to first introduce disability, identity and ageing to set the stage for the other aspects of ageing with a long-term disability may have been preferable. While most authors identify who is being quoted, some do not. Consistency in the presentation of vital demographics of informants, including age, gender and disability, following quotes would add meaning to the reference. Nevertheless, these minor suggestions in no way diminish the book's value in encompassing the discourse of ageing with a long-term disability.

The influence of this book should extend beyond the gaps in knowledge that it fills to stimulate reflection and advocacy about these issues. Undoubtedly, this text will serve as a leverage point from which further research and policy platforms may be developed. The ability to achieve and maintain independence and autonomy in spite of fundamental daily dependence and interdependence on support and services requires further detail and dialogue. The awareness that successful ageing with a long-term disability or illness involves optimising adaptations, enhancing capacities and compensating for losses or limitations across the lifecourse compels greater awareness. This is an important book that deserves a readership from political sociology, to gerontology and disability studies. 